\title{
Factors affecting the pre-filling probability of water storage tanks
}

\author{
G. Becciu \& A. Raimondi \\ Politecnico di Milano, Italy
}

\begin{abstract}
Water Storage tanks have proved to be effective for runoff control in drainage systems. Standard design procedures follow an event-based approach: a single flood at a time is considered and tank is assumed completely empty at the beginning of its filling. The possibility of pre-filling from previous events is then neglected and underestimation of storage volume can occur. In this paper an analytical probabilistic approach to estimate the probability of pre-filling is presented and effects due to outflow rate and storage volume are investigated. Derived formulas are validated by their application to a case study.

Keywords: water storage tanks, analytical probabilistic approach, pre-filling probability.
\end{abstract}

\section{Introduction}

In last decades the significant and rapid increase of impervious surfaces has made more frequent the overload of drainage systems, with uncontrolled polluted spills into receivers. Runoff control for reduction of combined and separated sewer overflow spills is typically accomplished through the implementation of storage tanks.

Although the proper modeling of these facilities should be based on the analysis of stochastic process of flood events, often an event-based approach is adopted for their design. A design (critical) rainfall, extracted from a recorded series or defined by a standard pattern, is used as input of a rainfall - runoff model to reproduce a flood hydrograph from which the needed minimum tank capacity is estimated. This flood event, although associated with a return period, is considered isolated from the whole stochastic process and the tank is assumed always empty at its beginning. Pre-filling from previous events is then neglected and an underestimation of the minimum storage size may occur. 
Approaches based on continuous simulation are more reliable and their results are usually more accurate. However, they are more complex and they may be difficult to apply due to the lack of rainfall data.

An alternative approach is the analytical probabilistic modeling (Guo and Adams [1-3]; Adams and Papa [4]), based on the derivation of the probability distribution functions of some characteristic parameters of the storage process from those of both the rainfall process and the drainage catchment. Taking advantage of some simplifying hypotheses, these approaches generally allow the development of relationships that combine the simplicity of event-based methods and the probabilistic reliability of continuous simulations. Their applications in literature, however, neglect the possibility of pre-filling from previous events or consider it only as an initial condition of storage (Becciu et al. [5]).

In this paper a procedure to fill this gap of application of analytical probabilistic modelling to storage tanks is proposed. Formulas for the estimation of pre-filling probability are derived and effects due to discharge rules and outflow rates on pre-filling probability are analysed and discussed. Application to a case study in Italy is presented. Finally, to test reliability of derived formulas and to investigate the effects of simplifying assumptions, results have been compared with those obtained with the continuous simulation approach.

\section{Modelling of storage tanks}

Independent rainfall events can be isolated from a continuous record of rainfalls defining a minimum interevent time, the so called InterEvent Time Definition (IETD) (USEPA [5]). If the interevent time is lower than IETD, two consecutive rainfalls are joined together into a single event, otherwise they are considered independent.

Meteorological input variables that most affect the modeling of storage tanks (rainfall depth, duration and interevent time) have been considered independent and exponentially distributed:

$$
\begin{gathered}
\mathrm{f}_{\mathrm{h}}=\xi \cdot \mathrm{e}^{-\xi \mathrm{h}} . \\
\mathrm{f}_{\theta}=\lambda \cdot \mathrm{e}^{-\lambda \theta} \\
\mathrm{f}_{\mathrm{d}}=\psi \cdot \mathrm{e}^{-\psi(\mathrm{d}-\text { IETD })}
\end{gathered}
$$

where $\xi=1 / \mu_{\mathrm{h}}, \lambda=1 / \mu_{\theta}, \psi=1 /\left(\mu_{\mathrm{d}}\right.$-IETD) and $\mu_{\mathrm{x}}$ are the expected value of random variable $\mathrm{x}$. In literature, this hypothesis has been often confirmed or considered acceptable in order to reduce the complexity of analytical derivation (Adams et al. [7], Eagleson [8], Bedient and Huber [9]).

Runoff volume has been defined as $\varphi$ (h-IA), where $\varphi$ is the runoff coefficient and IA (Initial Abstraction) is the loss for evaporation and depression storage at the beginning of the event. Hydrological losses have been averaged on rainfall duration (fig.2). A uniform loss equal to $(1-\varphi)(\mathrm{h}-\mathrm{IA})$, occurring after the initial depression storages have been filled, has been considered. 


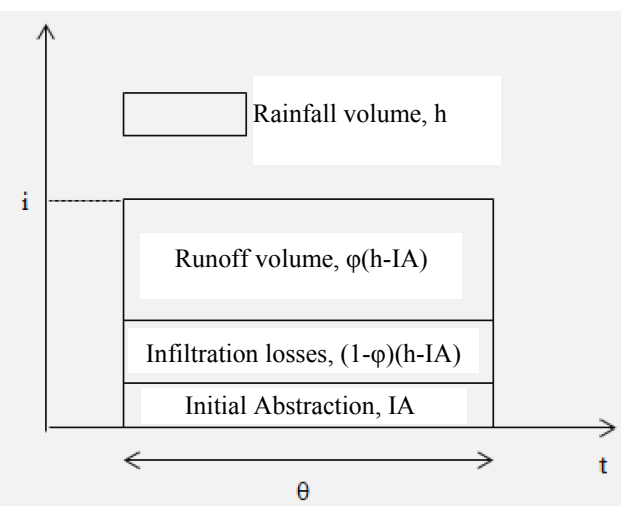

Figure 1: Hydrological losses averaged on rainfall duration.

Rainfall-runoff transformation has been neglected and net rainfall intensities have been considered as inflow rates in the tank. This hypothesis can be reliable for small catchments where runoffs can be assumed approximately proportional to rainfall intensities. Incoming hydrographs have been assumed rectangular, neglecting the temporal distribution of rainfall intensity within a storm event. This can be considered acceptable, being storage processes mainly driven by volumes than by discharges. Outflow rate from the tank has been also assumed constant.

A pair of flood events at a time has been considered, assuming that pre-filling can be due to one previous event only. This assumption can be acceptable when enough long IETD and high outflow rates are considered (Raimondi [10]). Two tank management rules have been considered, according to the more frequent strategies of discharge control:

Management rule A: the storage tank is emptied, with a constant outflow (q), starting as soon as it begins to fill. Considering rectangular events with inflow greater than outflow, this means soon after the beginning of each event (fig.2). It is a typical with on-line storage basins.

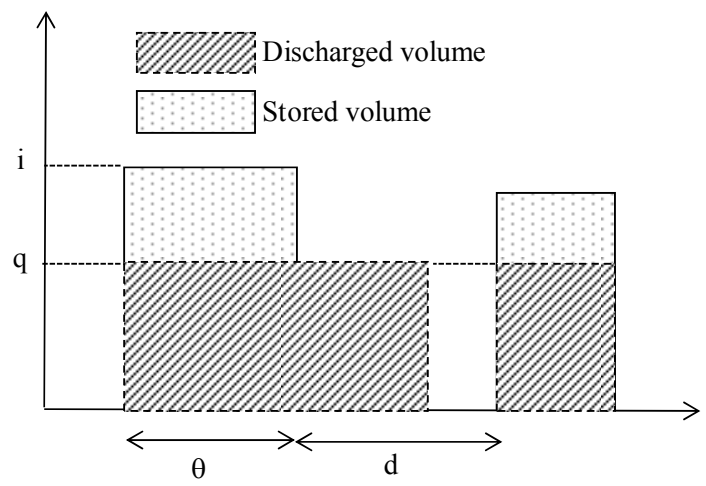

Figure 2: $\quad$ Management rule A. 
Management rule B: the constant outflow (q) starts after the end of each event. The outflow goes on until the tank is empty or a next event begins (fig.3). Management rule B can be used in Real Time Control (RTC) applications, when is necessary to temporarily retain a certain volume to reduce the risk of downstream system overload.

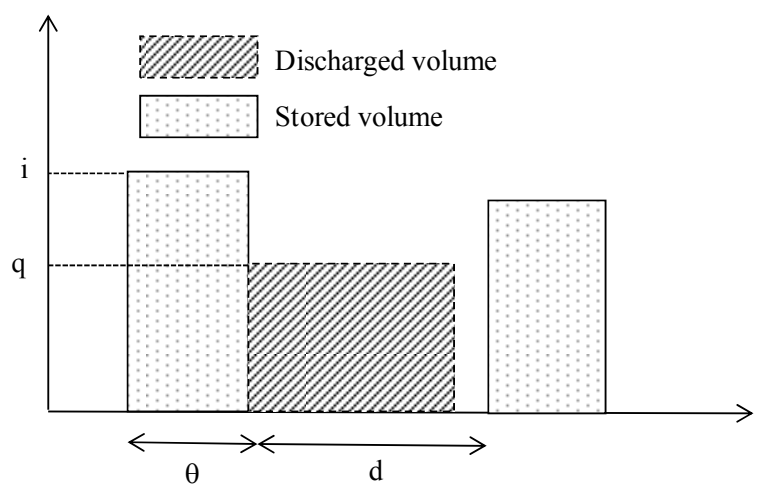

Figure 3: $\quad$ Management rule B.

\section{Pre-filling probability}

With both rules, it is possible to have a non-zero probability of a pre-filling volume greater then a certain percentage $\alpha$ of the tank storage capacity $\mathrm{w}_{0}$ only if the maximum emptying time results greater than the minimum interevent time (IETD). If volumes and flow rates are expressed per unit of effective catchment area $\varphi S$, as it will be in all the following formulas, this condition leads to the equivalent inequalities:

$$
\begin{gathered}
w_{0}(1-\alpha) / q>\text { IETD } \\
q<q_{M}=w_{0}(1-\alpha) / \text { IETD }
\end{gathered}
$$

must hold. Expressions for the estimation of the pre-filling probability are then derived with reference to the two above described management rules.

Management rule A

Pre-filling volume $\mathrm{w}_{\mathrm{pr}}$ can be expressed as:

$$
w_{p r}=\left\{\begin{array}{lll}
h-I A-q \theta-q d & & h-I A-q \theta<w_{0} \cup h-I A-q \theta-q d>0 \\
w_{0}-q d & \text { if } & h-I A-q \theta \geq w_{0} \cup w_{0}-q d>0 \\
0 & & \text { otherwise }
\end{array}\right.
$$


Combining conditions from eqn (6) together and using PDFs of rainfall depth, duration and interevent time $(\mathrm{h}, \theta, \mathrm{d})$, the probability that pre-filling volume exceeds a certain percentage $\alpha$ of the tank storage capacity results:

$$
\begin{aligned}
& P_{A}\left(\alpha w_{0}\right)=\operatorname{Prob}_{A}\left(w_{p r}>\alpha w_{0}\right)= \\
& =\int_{\theta=0}^{\infty} f_{\theta} d \theta \int_{h=q(\theta+I E T D)+I A}^{w_{0}+q \theta+I A} \int_{h} d h \int_{d=I E T D}^{(h-I A) / q-\theta} f_{d} d d+\int_{\theta=0}^{\infty} f_{\theta} d \theta \int_{h=w_{0}+q \theta+I A}^{\infty} f_{h} d h \int_{d=I E T D}^{w_{0} / q} f_{d} d d= \\
& =e^{-\xi I A} \frac{(1-\beta)}{\left(1+q^{*}\right)}\left\{e^{-\xi q I E T D-\psi I E T D}-e^{-\frac{\xi w_{0}}{\beta}}\right\}
\end{aligned}
$$

where $q^{*}=q \frac{\mu_{\theta}}{\mu_{h}}=q \frac{\xi}{\lambda}$ and $\beta=\frac{q \xi}{\psi+q \xi}$.

If $\alpha=0$, that for a prefilling volume greater than zero, eqn (7) becomes:

$$
\begin{aligned}
& P_{A}(0)=\operatorname{Prob}_{A}\left(w_{p r}>0\right)= \\
& =\int_{\theta=0}^{\infty} f_{\theta} d \theta \int_{h=q(\theta+I E T D)+I A}^{w_{0}+q \theta+I A} f_{h} d h \int_{d=I E T D}^{(h-I A) / q-\theta} f_{d} d d+\int_{\theta=0}^{\infty} f_{\theta} d \theta \int_{h=w_{0}+q \theta+I A}^{\infty} f_{h} d h \int_{d=I E T D}^{w_{0} / q} f_{d} d d= \\
& =e^{-\xi I A} \frac{(1-\beta)}{\left(1+q^{*}\right)}\left\{e^{-\xi q I E T D-\psi I E T D}-e^{-\frac{\xi w}{\beta}}\right\}
\end{aligned}
$$

Management rule B

Pre-filling volume $\mathrm{w}_{\mathrm{pr}}$ can be expressed as:

$$
w_{p r}= \begin{cases}h-I A-q d & h-I A<w_{0} \cup h-I A-q d>0 \\ w_{0}-q d & \text { if } \quad h-I A \geq w_{0} \cup w_{0}-q d>0 \\ 0 & \text { otherwise }\end{cases}
$$

Combining conditions from eqn (9) together and using PDFs of rainfall depth and interevent time $(\mathrm{h}, \mathrm{d})$, the probability that pre-filling volume exceeds a certain percentage of the storage volume $\left(\alpha \mathrm{w}_{0}\right)$ results:

$$
\begin{aligned}
& P_{B}\left(\alpha w_{0}\right)=\operatorname{Prob}_{B}\left(w_{p r}>\alpha w_{0}\right) \\
& =\int_{h=q I E T D+I A+\alpha w_{0}}^{w_{0}+I A} f_{h} d h \int_{d=I E T D}^{\left(h-I A-\alpha w_{0}\right) / q} f_{d} d d+\int_{h=w_{0}+I A}^{\infty} f_{h} d h \int_{d=I E T D}^{w_{0}(1-\alpha) / q} f_{d} d d= \\
& =e^{-\xi I A}(1-\beta)\left\{e^{-\xi q I E T D-\xi \alpha w_{0}-\psi I E T D}-e^{w_{0}\left[\frac{\psi(\alpha-1)}{q}-\xi\right]}\right\}
\end{aligned}
$$


As it can be noted, in this case the probability of pre-filling depends doesn't depend on rainfall duration PDFs, because the emptying process starts only after the rainfall is ended. If $\alpha=0$, eqn (10) becomes:

$$
\begin{aligned}
& P_{B}(0)=\operatorname{Prob}_{B}\left(w_{p r}>0\right)=\int_{h=q I E T D+I A}^{w_{0}+I A} f_{h} d h \int_{d=I E T D}^{(h-I A) / q} f_{d} d d+\int_{h=w_{0}+I A}^{\infty} f_{h} d h \int_{d=I E T D}^{w_{0} / q} f_{d} d d= \\
& =e^{-\xi I A}(1-\beta)\left\{e^{-\xi q I E T D-\psi I E T D}-e^{-\frac{\xi w_{0}}{\beta}}\right\}
\end{aligned}
$$

\section{Effects due to outflow rate and storage volume}

For both management rules, results depend on both minimum interevent time (IETD) and Initial Abstraction (IA). Resulting formulas are similar, except for the independence from rainfall duration with management rule B. However, comparing eqns (7) and (10), it results:

$$
P_{A}\left(w_{p r}>\alpha w_{0}\right)=\frac{P_{B}\left(w_{p r}>\alpha w_{0}\right)}{1+q^{*}}
$$

that is, as expected, pre-filling probability is lower with rule A than with rule $\mathrm{B}$, but this difference decreases with the outflow rate $\mathrm{q}$. As q tends to zero, $\mathrm{P}_{\mathrm{A}}$ and $\mathrm{P}_{\mathrm{B}}$ tend to the same limit value (fig. 4):

$$
\lim _{q=0} P_{A}\left(w_{p r}>\alpha w_{0}\right)=\lim _{q=0} P_{B}\left(w_{p r}>\alpha w_{0}\right)=P_{0}=e^{-\xi\left(I A+\alpha w_{0}\right)}
$$

while, considering eqn (5), as q tends to $\mathrm{q}_{\mathrm{M}}$, both $\mathrm{P}_{\mathrm{A}}$ and $\mathrm{P}_{\mathrm{B}}$ tend to zero (fig. 4).

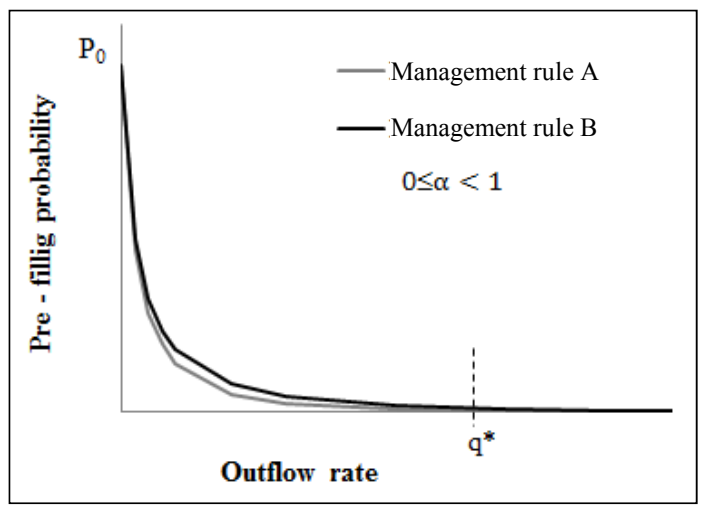

Figure 4: Pre-filling probability vs. outflow rate. 
Considering the effect of storage volume $w_{0}$, from eqn (4) descends that the pre-filling probability is, for both management rules, greater than zero for values greater than $w_{0}=w^{*}=q I E T D /(1-\alpha)$. For $0<\alpha<1$, both $\mathrm{P}_{\mathrm{A}}$ and $\mathrm{P}_{\mathrm{B}}$ tends to zero as $w_{0}$ tends to infinity (fig.5). A maximum value is reached for $w_{0}=w_{0 M}$ :

$$
\begin{aligned}
& \max _{w_{0}} P_{A}=P_{A}\left(w_{0 M}\right)=P_{A M}=\frac{(1-\beta)}{\left(1+q^{*}\right)} e^{-\xi I A-\frac{(\xi q+\psi) I E T D}{1-\delta}} \cdot\left[e^{-\frac{\delta \ln \delta}{1-\delta}}-e^{-\frac{\ln \delta}{1-\delta}}\right] \\
& \max _{w_{0}} P_{B}=P_{B}\left(w_{0 M}\right)=P_{B M}=(1-\beta) e^{-\xi I A-\frac{(\xi q+\psi) I E T D}{1-\delta}} \cdot\left[e^{-\frac{\delta \ln \delta}{1-\delta}}-e^{-\frac{\ln \delta}{1-\delta}}\right]
\end{aligned}
$$

with

$$
\begin{gathered}
w_{0 M}=\frac{(\xi q+\psi) I E T D-\ln \delta}{\xi \alpha\left(\frac{1}{\delta}-1\right)} \\
\delta=\frac{\alpha \beta}{1-\alpha(1-\beta)}
\end{gathered}
$$

For $\alpha=0$, as as $w_{0}$ tends to infinity the pre-filling probabilities tend to the constant values :

$$
\begin{aligned}
& \lim _{w_{0}=\infty} P_{A}=P_{\lim , A}=\frac{(1-\beta)}{\left(1+q^{*}\right)} e^{-\xi I A+(\xi q+\psi) I E T D} \\
& \lim _{w_{0}=\infty} P_{B}=P_{\lim , B}=(1-\beta) e^{-\xi I A+(\xi q+\psi) I E T D}
\end{aligned}
$$

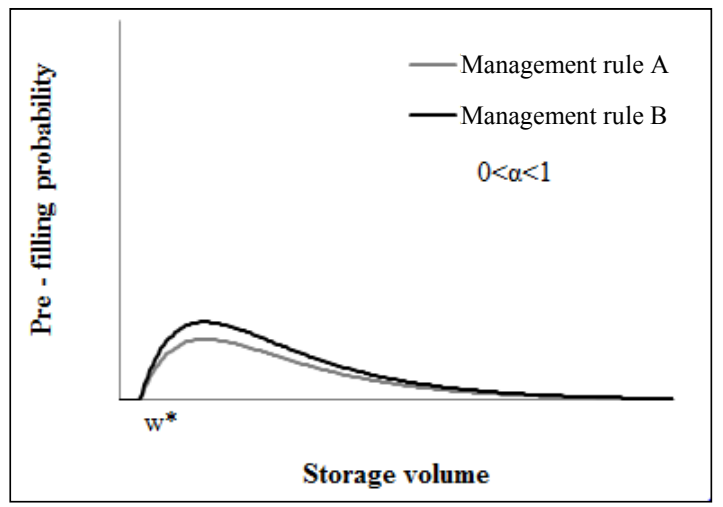

Figure 5: Pre-filling probability varying storage volume for $0<\alpha<1$. 


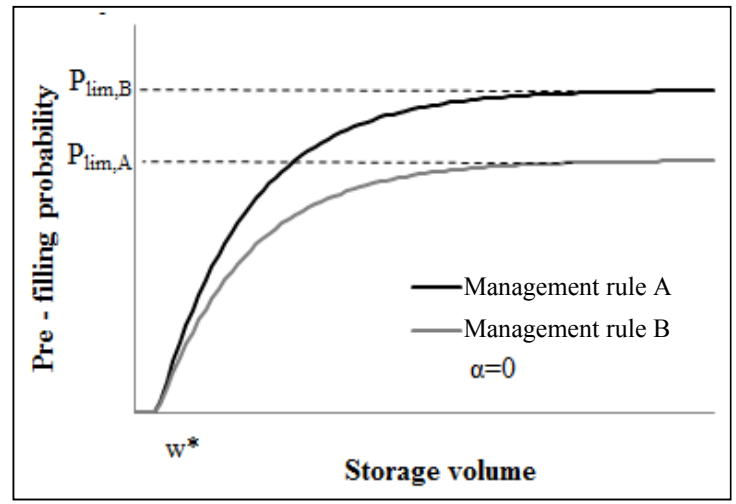

Figure 6: $\quad$ Pre-filling probability varying storage volume for $\alpha=0$.

\section{Case study}

Formulas for the estimation of the pre-filling probability have been applied using a series of rainfall events recorded in the period 1991-2005 at the raingauge of Monviso in the city of Milano, Italy. An IETD $=10$ hours has been selected, identifying $\mathrm{N}=1647$ independent rainfall events (Raimondi [10]), and an Initial Abstraction IA $=2 \mathrm{~mm}$ was assumed. Mean, standard deviation and coefficient of variation of rainfall depth, duration and interevent time of recorded series of events are reported in table 1.

As can be deduced by the coefficient of variations, only the hypothesis of exponential distribution of rainfall duration seems correct. Several authors already highlighted that other probability distributions, for example the Weibull, should be considered for the other rainfall characteristics (see e.g. Bacchi et al. [11]). However, to test the proposed formulas, following other studies in literature, an exponential distribution was fitted to all the samples of rainfall characteristics. A certain bias in results is then expected.

Table 1: Mean, standard deviation and coefficient of variation of rainfall depth, duration and intervent time recorded in the period 1991-2005 at raingauge of Monviso, Milano (Italy) for IETD $=10$ hours.

\begin{tabular}{|c|l|c|}
\hline$\mu_{\mathrm{h}}[\mathrm{mm}]=18.49$ & $\sigma_{\mathrm{h}}[\mathrm{mm}]=21.33$ & $\mathrm{~V}_{\mathrm{h}}[-]=1.15$ \\
$\mu_{\theta}[$ hours $]=14.37$ & $\sigma_{\theta}[$ hours $]=14.81$ & $\mathrm{~V}_{\theta}[-]=1.03$ \\
$\mu_{\mathrm{d}}[$ hours $]=172.81$ & $\sigma_{\mathrm{d}}[$ hours $]=223.89$ & $\mathrm{~V}_{\mathrm{d}}[-]=1.30$ \\
\hline
\end{tabular}

To test the other simplifying hypothesis of independence of rainfall variables, correlation coefficients have been calculated (table 3). While rainfall depth and interevent time, as well as rainfall duration and interevent time, are only weakly correlated, rainfall depth and duration could not be assumed as independent. 
Table 2: $\quad$ Correlation indexes among rainfall variables for IETD=10 hours.

\begin{tabular}{|cc|}
\hline \multicolumn{2}{|c|}{ IETD = 10 hours } \\
\hline$\rho_{\mathrm{h}, \theta}$ & 0,62 \\
$\rho_{\mathrm{d}, \theta}$ & 0,11 \\
$\rho_{\mathrm{h}, \mathrm{d}}$ & 0,11 \\
\hline
\end{tabular}

Also in this case, the assumption of independence in the proposed approach will cause a bias in the results..

To test the accuracy of proposed formulas, also considering the simplifications adopted in the probability scheme, frequencies of pre-filling have been calculated assuming the recorded series of rainfall events as a series of rectangular floods incoming in a storage tank. Storage volumes ranging between 50 and $600 \mathrm{~m}^{3} / \mathrm{ha}_{\text {imp }}$ and outflow rates of 1 and $3 \mathrm{l} /\left(\mathrm{s} \cdot \mathrm{ha}_{\text {imp }}\right)$ have been considered. Figures 7 and 8 show results obtained with $\alpha=0$ for both management rule $\mathrm{A}$ and $\mathrm{B}$.

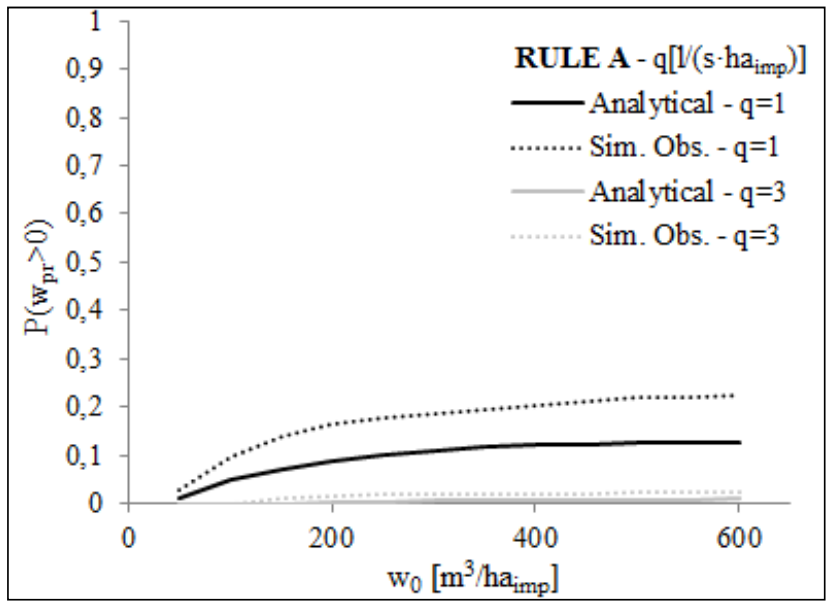

Figure 7: $\quad$ Pre-filling CDFs. IETD $=10$ hours and $\alpha=0$. Management rule A.

As can be seen, the comparison between pre-filling probabilities estimated with analytical formulas (8) and (11) and frequencies calculated by continuous simulation of the recorded series highlights a clear bias. The proposed formulas underestimate the probability of pre-filling, especially for lower outflow rates. This happens for both the management rules, but it is more significant for rule B. As expected, the simplifying hypotheses of uncorrelated exponential random variables play a significant role in the accuracy of results. 


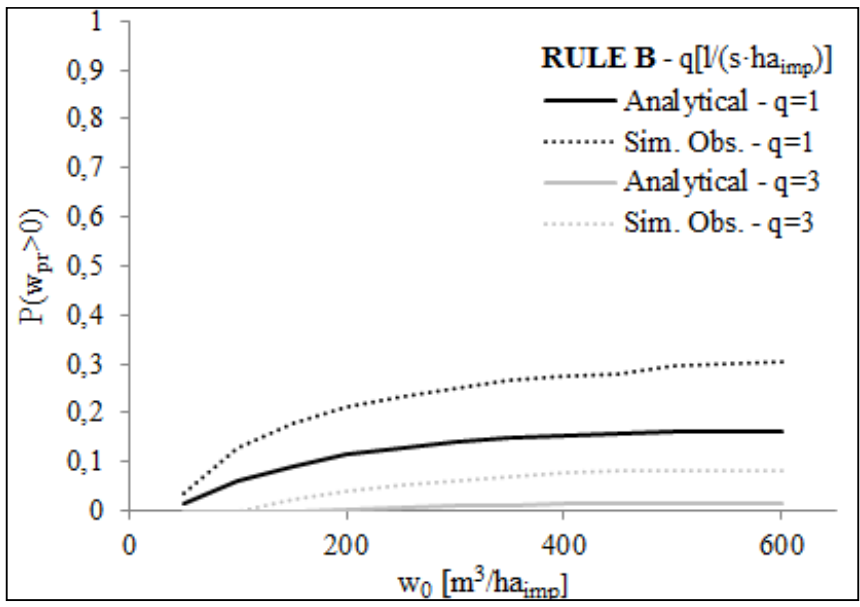

Figure 8: $\quad$ Pre-filling CDFs. IETD $=10$ hours and $\alpha=0$. Management rule B.

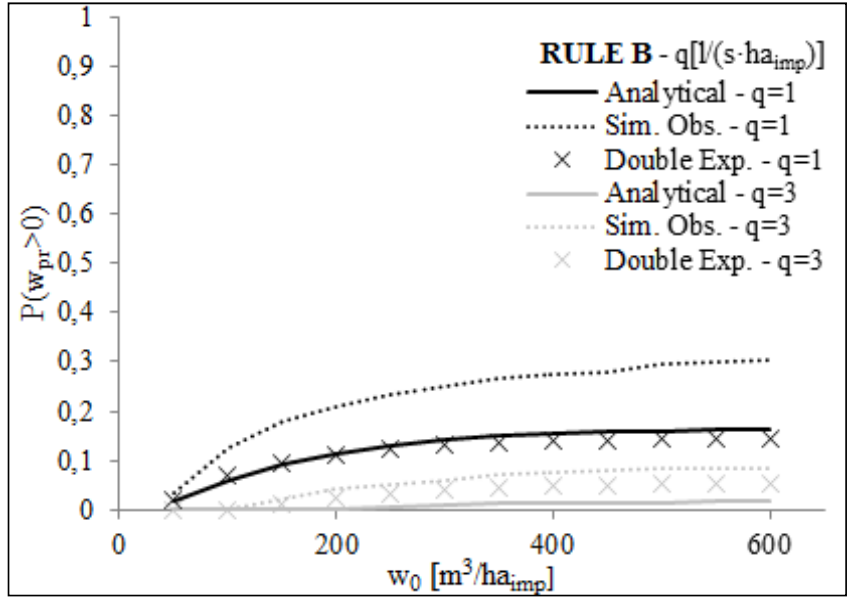

Figure 9: $\quad$ Pre-filling CDFs. IETD $=10$ hours and $\alpha=0$. Management rule B.

It has to be observed that with management rule B, the tank starts emptying only at the end of rainfall event, making the probability of pre-filling independent from rainfall duration, as can be seen in eqn. from (9) to (11).

Considering that rainfall depth and interevent time are only weakly correlated (table 3), the hypothesis of independent variables does not affect very much results. In this case, the underestimation is mainly due to the assumption of exponential distributions. To test how much this assumption influences results, another distribution was assumed for the rainfall depth and the interevent time, while the exponential distribution was maintained for the rainfall duration. Analytical formulas derived in this way are more complicated and have not 
reported. As can be seen in fig.9, the use of a double-exponential distribution improves the accuracy of results from analytical formulas, especially for higher outflow rates.

For lower outflow rates the persisting bias may be due to the modeling of only a pair of events at a time. In this case the pre-filling volume may be caused by more than one previous event. Future analysis will be focused also on this aspect.

\section{Conclusions}

Storage tanks are effective tools for runoff control. Their capacity must be carefully estimate to avoid uncontrolled spills into receivers. In some cases prefilling of storage volume from previous storms can occur and the capacity of the facility can be underestimated.

An analytical probabilistic method was proposed, useful to perform a simple and direct estimation of the probability of pre-filling for two difference tank management rules. Derived expressions depend on the stochastic process of the rainfall, storage volume and outflow rate. IETD and IA are also taken into account.

Proposed formulas and application to a case study show that pre-filling can't be neglected for low outflow rate, e.g. in the case of strict discharge limits in the downstream water system or of infiltration basins with low permeability soils. Although application to the case study shows that accuracy is not completely satisfactory, due to simplifying hypotheses, the simple form of proposed formulas make them useful for most engineering applications.

\section{References}

[1] Guo Y., Adams B. J., Hydrologic analysis of urban catchments with eventbased probabilistic models. 1. Runoff volume, Water Resources Research, vol. 34 (12), 3421-3443, 1998a.

[2] Guo Y., Adams B. J., Hydrologic analysis of urban catchments with eventbased probabilistic models. 2. Peak discharge rate, Water Resources Research, vol. 34 (12), 3421-3443, 1998 b.

[3] Guo Y., Adams B. J., An analytical probabilistic approach to sizing flood control detention facilities, Water Resources Research, vol. 53 (8), 24572468, 1999.

[4] Adams, B.J., Papa, F., Urban Stormwater Management Planning with analytical probabilistic models, John Wiley \& Sons, New York, USA, 1999.

[5] Becciu G., Paoletti A., Raimondi A., Sanfilippo U., Effects of management rules on pre-filling of stormwater detention facilities, 12th International Conference on Urban Drainage, Porto Alegre/Brazil, 2011. 
[6] U.S. Environmental Protection Agency (USEPA), Methodology for analysis of detention basins for control of urban runoff quality, EPA440/587-001, Washington, D.C., 1986.

[7] Adams, B.J., H.G. Fraser, C.D.D. Howard, and M.S. Hanafy, Meteorological data analysis for drainage system design, J. Environ. Eng., 112(5), 827-848, 1986.

[8] Eagleson, P.S., Climate, soil and vegetation. The distribution of annual precipitation derived from observed storm sequences, Water Resources Research, 14(5), 713-721, 1978.

[9] Bedient P. B. and Huber W.C., Hydrology and floodplain analysis, 2nd Ed., Addison Wesley, New York, USA, 1992.

[10] Raimondi A., Analytical probabilistic modeling of storage basins for stormwater control, $\mathrm{PhD}$ thesis, Politecnico di Milano, 2012.

[11] Bacchi B., Balistrocchi M., Grossi G. Proposal of a semi-probabilistic approach for storage facility design, Urban Water Journal, Vol. 5, No 3, pp. 195-208, 2008. 